\title{
Revisiones Sistemáticas Exploratorias
}

\section{Scoping review}

\section{Rocío Manchado Garabito}

Servicio de Salud Pública del Área 8.

Consejería de Sanidad de la Comunidad de Madrid. España.

\section{Sonia Tamames Gómez}

Servicio del Observatorio de Salud Pública

Dirección General de Salud Pública e Investigación, Desarrollo e Innovación

Consejería de Sanidad de la Junta de Castilla y León. Valladolid. España.

\section{María López González}

Facultad de Farmacia. Universidad Complutense. Madrid. España.

\section{Laura Mohedano Macías}

Subdirección General de Programas Internacionales de Investigación y

Relaciones Institucionales. Instituto de Salud Carlos III.

Ministerio de Ciencia e Innovación. Madrid. España.

\section{Marcelo D'Agostino}

Area de Gestión del Conocimiento y Comunicación. Organización

Panamericana de la Salud - Organización Mundial de la Salud (OPS/OMS).

Washington DC, USA.

\section{Jorge Veiga de Cabo}

Escuela Nacional de Medicina del Trabajo. Instituto de Salud Carlos III.

Ministerio de Ciencia e Innovación. Madrid. España.

\section{Correspondencia:}

Rocío Manchado Garabito

Servicio de Salud Pública del Área 8

Consejería de Sanidad de la Comunidad de Madrid.

Avda. de Leganés, 25

28925 ALCORCÓN (Madrid). España.

Tel: 916210090. Fax: 916100527

e-mai: rmanchado.hcsc@salud.madrid.org

\section{Resumen}

Debido al gran avance que se ha producido en los últimos años en el ámbito de la investigación científica, no sólo disponemos cada vez de más información si no que esta es a su vez más compleja. Las revisiones exploratorias permiten sintetizar la evidencia existente respecto a un tema en salud incorporando diferentes diseños de estudio, intervenciones y medidas de impacto con el fin de generar nuevas hipótesis, líneas de investigación o proponer métodos de trabajo más adecuados para futuras investigaciones. Esto es posible aplicando criterios de evaluación de la calidad de los documentos encontrados y del grado de evidencia científica.

(Med Segur Trab (Internet) 2009; 55 (216): 12-19)

Palabras clave: metodología, revision sistemática, revisión sistemática exploratoria

Abstract

Due to the great progress that has occurred in recent years in the field of scientific research, the information is increasing not just in terms of amount but also in complexity. The scoping reviews allow to synthesize the existing evidence regarding a health issue, incorporating different study designs, interventions 
and measures of impact in order to generate new hypotheses, lines of research or to propose most appropriate working methods for future research. This is possible by applying criteria for assessing the quality of the documents found and the levels of scientific evidence.

(Med Segur Trab (Internet) 2009; 55 (216): 12-19)

Key words: Methods, systematic review, scoping review 


\section{ANTECEDENTES}

Debido al gran avance que se ha producido en los últimos años en el ámbito de la investigación científica y en el uso del Internet, no sólo disponemos cada vez de más información, si no que esta es a su vez más compleja y se representa en diferentes formatos, convencionales (fuentes de información clásicas y bases de datos) y no convencionales (Blogs, Wikis, Redes Sociales) en donde el lector participa agregando valor a los contenidos (Web 2.0). Tradicionalmente, la evidencia se ha sintetizando de forma objetiva y científica mediante revisiones sistemáticas con el fin de contestar a una hipótesis concreta. En el caso de estudios con resultados cuantitativos, estos podían combinarse para lograr un estimador más preciso utilizando técnicas de metanálisis. Pero esta metodología puede resultar insuficiente cuando se trata de evaluar intervenciones complejas $^{1}$, en estos casos, puede ser interesante realizar una revisión sistemática exploratoria que nos de una idea de qué se ha hecho y de qué manera, permitiendo generar hipótesis e identificar aquellos aspectos que pueden ser estudiados con más detalle posteriormente. La mayoría de los elementos que forman parte de la metodología de la revisión sistemática tradicional son válidos para la revisión sistemática exploratoria, si bien es necesario comentar algunos aspectos que por su propio diseño son algo diferentes. Estas revisiones exploratorias se emplean con frecuencia para la elaboración de informes técnicos, que suele ser la herramienta más utilizada a la hora de la toma de decisiones en el ámbito de la Salud Laboral.

Los principales objetivos que perseguimos en una revisión sistemática, los podemos resumir en:

- Evaluar la calidad y metodología empleadas en las investigaciones realizadas en una determinada área de conocimiento.

- Sintetizar la evidencia científica.

- Servir de utilidad en la toma de decisiones.

Clasificación de los documentos de revisión:

Como se ha explicado en la introducción, existen diferentes tipo de revisiones sistemática de la literatura científica, así, podemos distinguir:

- Revisión sistemática: son trabajos que resumen de forma sistemática la evidencia científica para estudiar una relación existente en el ámbito de la salud contestando a una pregunta concreta.

- Meta-análisis: técnica de síntesis cuantitativa de resultados de investigaciones primarias con el objeto de proporcionar estimaciones más precisas que las que se desprenden de los estudios aislados incluidos en la revisión. Como dice Gene Vidrio Glass ${ }^{2-4}$ se trataría del análisis estadístico de una gran colección de resultados de trabajos individuales con el propósito de integrar los hallazgos obtenidos.

- Revisión Sistemática Exploratoria: síntesis de la evidencia sobre un tema relacionado con la salud que describe el conocimiento existente sobre el mismo. Sirven para generar hipótesis, establecer líneas de investigación, o como base para la elaboración de informes técnicos. Tabla $1^{5}$.

- Informe técnico: documento elaborado por expertos que seleccionan según su criterio la evidencia que responde a una pregunta determinada sobre un problema de salud. Suelen ser estudios basados habitualmente en revisiones sistemáticas (con o sin meta-análisis), otros informes técnicos, juicios de expertos u otros recursos de información. 
Tabla 1. Diferencias entre la revisión sistemática clásica y la revisión sistemática exploratoria.

\begin{tabular}{lll}
\hline Tipo de Estudio & Revisión sistemática clásica & Revisión sistemática exploratoria \\
\hline Hipótesis & Contesta a una pregunta concreta & Describe el conocimiento existente \\
Objetivos & Una intervención & Más de una intervención \\
Estudios incluidos & Cualitativos y cuantitativos & Todo tipo de estudios \\
Resultados & Una medida de impacto & Diferentes medidas de impacto \\
Criterios inclusión & Selección por calidad & Selección por relevancia \\
\hline
\end{tabular}

\section{LA REVISIÓN SISTEMÁTICA EXPLORATORIA}

Como se ha mencionado anteriormente, se trata de un tipo de revisión sistemática que se caracteriza por una serie de peculiaridades que permiten explorar no sólo qué se ha hecho sobre un tema, también quién, dónde y de qué manera. A partir de los resultados obtenidos pueden generarse hipótesis sobre futuras preguntas de investigación y proponerse ámbitos de estudio que no están suficientemente desarrollados. En este artículo hemos descrito el protocolo a seguir para elaborar una revisión sistemática exploratoria de calidad, incluyento aquellos elementos que son propios de esta. Tabla 2.

Tabla 2. Diseño del protocolo de una revisión sistemática exploratoria.

\begin{tabular}{l} 
Introducción \\
\hline Pregunta de estudio \\
Objetivo \\
\hline
\end{tabular}

\section{Metodología}

Criterios de inclusión:

Periodo de estudio

Idioma

Otros: población de estudio, área geográfica,

etc

Tipo de documentos incluidos

Identificar las fuentes de información y fecha de la última búsqueda.

Establecer la estrategia de búsqueda

(incluir los límites aplicados)

Selección y clasificación de los estudios

Definición de las variables de estudio.

Extracción de datos.

\begin{tabular}{ll} 
Resultados & \\
\hline Resumen del número de artículos obtenidos en cada fase. & $\begin{array}{l}\text { Artículos incluidos en la revisión } \\
\text { Artículos excluidos. Causas } \\
\text { Diagrama de flujo }\end{array}$
\end{tabular}

Análisis de la extracción de datos. Análisis bibliométrico

Conclusiones y recomendaciones 


\section{INTRODUCCIÓN}

Breve presentación del marco conceptual en el que se exponen tanto los conocimientos más consolidados del tema de interés como aquellos elementos que justifican una investigación más profunda respecto a algún área en concreto. Deben incluirse la pregunta de estudio y los objetivos en el último párrafo, aunque estos también pueden aparecer en otro apartado independiente. En el caso de las revisiones sistemáticas exploratorias y como se ha comentado con anterioridad, el objetivo es describir qué investigaciones se han realizado en un determinado área del conocimiento pudiéndose tener en cuenta distintos tipos de estudio, intervenciones y medidas de impacto.

\section{METODOLOGÍA}

Es diseño del protocolo debe aparecer descrito de forma detallada presentando todos y cada uno de los elementos que se han tenido en cuenta.

\section{Criterios de inclusión}

Para seleccionar qué documentos forman parte de la revisión se establecen una serie de criterios referentes tanto al documento (año de publicación, idioma, ámbito geográfico) como al contenido del mismo (tipo de diseño, temática etc) que se considera que en su conjunto contestan a la pregunta de estudio.

2. Identificar las fuentes de información y establecer la estrategia de búsqueda

El siguiente paso será identificar aquellas fuentes de información más adecuadas, en general, siempre que se trate de estudios de ciencias de la salud, la mayoría de la información útil puede encontrarse en bases de Datos Biomédicas (Cochrane Library, MEDLINE, LILACS, IBECS, IME, EMBASE, CIS-DOC, CUIDEN, etc), aunque en ocasiones, por requerimiento de la temática de la revisión sistemática exploratoria y de los objetivos de la misma, puede ser recomendable recurrir a otras fuentes de información no indexadas, generalmente denominadas 'literatura gris' (informes técnicos, consulta a expertos o páginas web de organismos e instituciones). Hay disponibles manuales y guías de consulta que orientan sobre la identificación de estas fuentes ${ }^{6-9}$.

3. Establecer la estrategia de búsqueda que incluya los límites aplicados.

La estrategia de búsqueda debe aparecer clara y detallada, existen guías de ayuda de gran utilidad a la hora de definirla ${ }^{10-11}$. Es conveniente utilizar los descriptores y calificadores de los "Thesauros" específicos de cada base de datos (MeSH y DeCS para MEDLINE, LILACS e IBECS, por ejemplo) ajustándose a los criterios de inclusión definidos en la metodología, lo que nos permite obtener una colección de artículos adecuada. Establecer correctamente la estrategia consiste en algo más que la mera utilización de términos que en un principio pueda parecer que se relacionan con la pregunta de estudio o con las palabras clave (las cuales casi nunca se identifican con los descriptores). Se trata de construir una fórmula que incluya los principales descriptores y calificadores que jerárquicamente se encuentran estructurados en el "Thesauro", permitiendo el mayor grado de precisión a la hora de localizar estos términos vinculados a los artículos incluidos en la base de datos bibliográfica que nos interesa que formen parte de la revisión. En definitiva, se trata de utilizar la misma estrategia de recuperación que la que se utilizó para clasificar los artículos en el momento en que fueron introducidos en la base de datos bibliográfica. Por esta razón, siempre que tengamos dudas, es preferible recurrir a documentalistas expertos que nos ayuden a establecer la estrategia correcta de recuperación, pues una gran parte del éxito de nuestro trabajo radica en la calidad, precisión y pertinencia de la colección de artículos resultantes de la búsqueda, y que en definitiva compondrán la base para los resultados del estudio.

En aquellos casos en los que sea necesario restringir la búsqueda por temas o áreas geográficas, se pueden aplicar filtros bibliográficos que eliminan el ruido o la cantidad 
de documentos no pertinentes (especificidad de la estrategia) a la vez que se gana en exhaustividad (sensibilidad de la estrategia), llegando al máximo número posible de estudios que contengan información referida a la pregunta de investigación. ${ }^{12-14}$

4. Selección y clasificación de los estudios.

Tras la obtención del primer listado de documentos, éstos deben ser clasificados. Una primera aproximación puede ser su catalogación como "pertinente o no pertinente", a partir de la información que se extrae del título y del resumen del artículo. Esta clasificación actuaría como primer filtro para la selección de los artículos de la colección que definitivamente pasarán a incluirse en la revisión sistemática. Esta simple acción puede facilitar mucho las tareas destinadas a la obtención del documento primario o artículo a texto completo, centrando los esfuerzos en localizar aquellos con mayor utilidad práctica.

Seguidamente se aplicarían los criterios de inclusión y exclusión, describiéndose claramente este proceso junto con el mecanismo de detección y eliminación de estudios duplicados. Por último, sobre las investigaciones seleccionadas puede realizarse una valoración del grado de interés del documento, por su calidad, grado de ajuste al tema de estudio o innovación, esta puntuación está dirigida a obtener un segundo nivel de priorización en la fase de obtención de los documentos primarios.

5. Definición de las variables de estudio.

En una revisión sistemática exploratoria, se recogen para cada uno de los estudios varios tipos de variables que deben aparecer definidas aclarando cualquier criterio que los autores hayan establecido.

- Variables de filiación del artículo o variables bibliométricas. Hacen referencia a aquellos aspectos del documento que sirven para filiarlo y enmarcarlo en un contexto sin entrar en detalles de contenido. A partir de estas variables se realiza el análisis bibliométrico. Tabla 3 .

Tabla 3. Variables bibliométricas

\begin{tabular}{|c|c|c|c|c|c|c|c|}
\hline $\begin{array}{l}\text { Base de datos } \\
\text { bibliográficas }\end{array}$ & $\begin{array}{c}\text { Año } \\
\text { publicación }\end{array}$ & Revista & $\begin{array}{c}\text { Tipo } \\
\text { de publicación }\end{array}$ & Autores & Institución & País & Idioma \\
\hline
\end{tabular}

- Variables para evaluar la calidad y grado de evidencia, a partir de las cuales puede valorarse la calidad de la metodología aplicada a la investigación así como su grado de evidencia. Justifican la inclusión o no de un documento en la revisión sistemática y sirven de base para las recomendaciones finales.

- Variables que recogen información del contenido del artículo. Estas vendrán determinadas por la pregunta de estudio y los objetivos, incluidas aquellas que sirvan para controlar sesgos y variabilidad entre estudios. Todos los tipos de intervenciones y medidas de resultado que vayan a contemplarse, deben especificarse con detalle ya que no todos los estudios trabajan sobre los mismos grupos de población y ámbito de aplicación. Esto permite identificar en la fase de análisis qué grupos se encuentran en mayor riesgo o sobre cuales determinadas actuaciones presentarán diferentes resultados.

6. Proceso de extracción de datos y confirmación de los mismos.

Consiste en la lectura crítica y obtención de la información necesaria de cada uno de los artículos seleccionados para completar las tablas con las variables descritas en el apartado anterior. Debe describirse el método seguido en el proceso de obtención de los datos y confirmación de los mismos. Vendrá especificado si se realiza revisión por pares, independiente y forma de realizar un desempate en caso de ser necesario.

\section{Comunicación de resultados}

En la fase de comunicación de los resultados se ponen de nuevo de manifiesto las particularidades descritas para las revisiones sistemáticas exploratorias con respecto a las 
clásicas. Si bien es necesario observar unos criterios de calidad en esta comunicación, también debe existir cierta flexibilidad para poder adecuar la comunicación de resultados a las singularidades de cada revisión. En este sentido, las directrices contenidas en la declaración QUOROM ${ }^{15}$ han sido sustituidas recientemente por la declaración PRISMA ${ }^{16}$.

En cualquier caso debe presentarse un diagrama de flujo en el que se refleje el número de documentos incluidos en la revisión, aquellos que han sido excluidos y las causas que han motivado tal exclusión.

La primera información que se obtiene parte del análisis bibliométrico de la colección recuperada, nos da una idea de la producción científica en torno al tema sobre el que se está trabajando (cuánto se investiga, quién lo hace, dónde se está produciendo la información, en que periodo, tendencias temporales, cuales son las instituciones que dedican más esfuerzos a investigar sobre el tema y qué revistas se hacen eco con mayor frecuencia de esta información). De este modo, y tras el análisis de las variables, se puede saber el grado de interés que puede despertar la hipótesis de estudio en la comunidad científica, si se ha trabajado de forma activa sobre ésta, si se trata de un tema obsoleto o por el contrario de una pregunta innovadora. Ayuda a identificar cuáles son las principales potencias productoras y qué revistas científicas pueden tener mayor interés para nosotros tanto para obtener información relevante como para identificar posibles canales que permitan mayor divulgación de los resultados obtenidos en la revisión sistemática.

El segundo paso será realizar un análisis crítico de la metodología seguida aplicando criterios de calidad a cada uno de los estudios incluidos. En este punto debe tenerse en cuenta que la variabilidad en el diseño es muy amplia en las revisiones exploratorias cuyo principal objetivo es saber qué se ha hecho, y por tanto se incluye un repertorio amplio. Los criterios de calidad establecidos en el protocolo sirven para discriminar qué investigaciones formarán parte de la revisión, desechando aquellas que se considera que no cumplen un mínimo de rigurosidad o no son adecuadas para contestar a la hipótesis.

El núcleo de la exposición consistirá en resumir los principales resultados encontrados, en función del área temática, grado de evidencia y relevancia hacia los diferentes actores que pudieran estar interesados: personal asistencial, usuarios, tomadores de decisiones, políticos o gestores. Debe comentarse igualmente la posibilidad de aparición de sesgos, las limitaciones, y como influirían ambos sobre los resultados obtenidos.

\section{Conclusiones y recomendaciones}

Finalmente, se presentarán las conclusiones y recomendaciones de acuerdo con los objetivos enunciados y la información obtenida en el proceso de revisión. Con carácter adicional, los autores podrían proponer un método más adecuado con el que generar evidencia científica de mayor calidad y más acorde con lo que se pretende estudiar en posteriores investigaciones.

\section{BIBLIOGRAFÍA}

1. Shepperd S, Lewin S, Straus S, Clarke M, Eccles MP, et al. (2009) Can We Systematically Review Studies That Evaluate Complex Interventions? PLoS Med 6(8): e1000086. doi:10.1371/journal.pmed.1000086.

2. Vidrio, G. V (1976). El análisis primario, secundario y meta-de la investigación. Educational Researcher, 5, 3-8.

3. Vidrio, G.V (1978). La integración de los resultados: El meta-análisis de la investigación. Revisión de la Investigación en Educación, 5, 351-379.

4. Vidrio, G. V, McGaw, B., \& Smith, M. L. (1981). Meta-análisis en la investigación social. Beverly Hills, CA: Sage Publications

5. Poth C, Ross S, Meta-analysis, systematic review, or scoping review? Comparing methodologies in educational research. http://ocs.sfu.ca/fedcan/index.php/csse2009/csse2009/paper/viewDownloadInterstitial/1867/552

6. Bojo Canales C, Fraga Medín C, Hernández Villegas S, Jaén casquero MB, Jiménez Planet V, Mohedanos Macías L, Novillo Ortiz A. Internet Visible e Invisible: búsqueda y selección de recursos de información en Ciencias de la Salud. Madrid. Instituto de Salud Carlos III; 2004. http://bvs.isciii.es/mono/pdf/BNCS_01.pdf. Consultado 1 de junio de 2009. 
7. Culebras J, García Lorenzo A, Gil A, Laborda L, Luengo Pérez LM, Moreno de Torres R, et al. Open Access: Gestión y acceso a la literatura científica. Grupo de Comunicación y Documentación Científica en Nutrición (CDC Nut-SENPE); 2007. http://www.senpe.com/grupos/senpe_publicaciones_cdcnut/openacces.pdf. Consultado 1 de junio de 2009.

8. Sanz-Valero, J, D`Agostino MJ, Castiel LD, Veiga de Cabo J. La iniciativa Open Access, una visión de conjunto. Med Segur Trab. 2007, (53); 207. 05-09. http://scielo.isciii.es/pdf/mesetra/v53n207/original2.pdf. Consultado 1 de junio de 2009.

9. http://infosaludlaboral.isciii.es

10. Wanden-Berghe C, Veiga de Cabo J, Sanz-Valero J, Perez de la Cruz A, Culebras J, García Lorenzo A, et al. De la pregunta de investigación a la ecuación de búsqueda bibliográfica: los Descriptores en Ciencias de la Nutrición. Madrid. Grupo de Comunicación y Documentación Científica en Nutrición (CDC Nut-SENPE); 2008. http://www. senpe.com/grupos/senpe_publicaciones_cdcnut/Libro2009senpe.pdf. Consultado 1 de junio de 2009.

11. http://www.fisterra.com

12. Arencibia Jorge R, Perezleo Solorzano L, Araujo Ruiz JA. Los filtros metodológicos como herramientas eficaces para la búsqueda de evidencias clínicas. ACIMED [revista en Internet]. 2004 [citado 4 feb 2008]; vol 12 (3): [aprox. 21 pantallas]. Disponible en: http://scielo.sld.cu/scielo.php?script=sci_arttext \& pid=S102494352004000300005 \& lng=es \& nrm=iso \& tlng=es.

13. Arranz M. Los filtros metodológicos y la Medicina Basada en la Evidencia (MBE). Pap Med. 2003;12(1):8-10.

14. Sanz-Valero Javier, Veiga de Cabo Jorge, Rojo-Alonso Carlos, D`Agostino Marcelo José, Wanden-Berghe Carmina, Espulgues Pellicer Josep Xavier et al. Los filtros metodológicos: aplicación a la búsqueda bibliográfica en la medicina del trabajo española. Med. segur. trab. [revista en la Internet]. 2008 Jun [citado 2009 Dic 28] ; 54(211): 75-83. Disponible en: http://scielo.isciii.es/scielo.php?script=sci_arttext\&pid=S0465546X2008000200009\&lng=es.

15. Moher D, Cook DJ, Eastwood S, Olkin I, Rennie D, et al. (1994) Improving the quality of reporting of metaanalysis of randomized controlled trials: The QUOROM statement. Lancet 354: 1896-1900.

16. Moher D, Liberati A, Tetzlaff J, Altman DG, The PRISMA Group (2009) Preferred Reporting Items for Systematic Reviews and Meta-Analyses: The PRISMA Statement. PLoS Med 6(7): e1000097. doi:10.1371/ journal.pmed.1000097

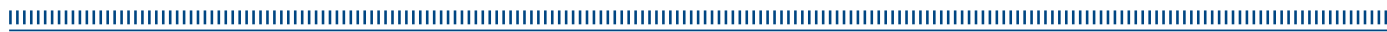

